\title{
DFT calculation for the electronic properties and quantum capacitance of pure and doped $\mathrm{Zr} 2 \mathrm{CO} 2$ as electrode of supercapacitors
}

\author{
Shuo $\mathrm{Xu}^{1}$, Shi-Jie Wang ${ }^{1}$, Li Xiao-Hong ${ }^{1}$, and Hong-Ling Cui ${ }^{1}$ \\ ${ }^{1}$ Henan University of Science and Technology
}

September 24, 2021

\begin{abstract}
Defect and doping are effective methods to modulate the physical and chemical properties of materials. In this report, we investigated the structural stability, electronic properties and quantum capacitance (Cdiff) of Zr2CO2 by changing the dopants of $\mathrm{Si}, \mathrm{Ge}, \mathrm{Sn}, \mathrm{N}, \mathrm{B}, \mathrm{S}$ and $\mathrm{F}$ in the substitutional site. The doping of F, N, and $\mathrm{S}$ atoms makes the system undergo the semiconductor-to-conductor transition, while the doping of $\mathrm{Si}, \mathrm{Ge}$, and $\mathrm{Sn}$ maintains the semiconductor characteristics. The Cdiff of the doped systems are further explored. The B-doped system can be used as cathode materials, while the systems doped by S, F, N, Sn atoms are promising anode materials of asymmetric supercapacitors, especially for the S-doped system. The improved Cdiff mainly originates from Fermi-level shifts and Fermi-Dirac distribution by the introduction of the dopant. The effect of temperature on Cdiff is further explored. The result indicates that the maximum Cdiff of the studied systems gradually decreases with the increasing temperature. Our investigation can provide useful theoretical basis for designing and developing the ideal electrode materials for supercapacitors.
\end{abstract}

\section{Hosted file}

quantum capacitance of pure and doped Zr2C02-IJQC.docx available at https://authorea.com/ users/435870/articles/538601-dft-calculation-for-the-electronic-properties-and-quantumcapacitance-of-pure-and-doped-zr2co2-as-electrode-of-supercapacitors 